Check for updates

Cite this: RSC Adv., 2017, 7, 49895

Received 7th September 2017

Accepted 14th October 2017

DOI: 10.1039/c7ra09999a

rsc.li/rsc-advances

\section{Establishment of a rapid and sensitive method based on recombinase polymerase amplification to detect mts90, a new molecular target of Mycobacterium tuberculosis $\uparrow$}

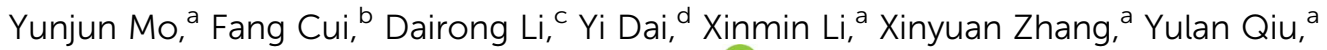
Yibing Yin, ${ }^{a}$ Xuemei Zhang ${ }^{a}$ and Wenchun Xu (D)*a

\begin{abstract}
Tuberculosis (TB) remains a significant challenge to public health, especially in developing countries. Failure in early diagnosis and lack of rapid and accurate diagnostic methods lead to ongoing prevalence and transmission of TB. Recently, the recombinase polymerase amplification (RPA) technique has made it possible to rapidly amplify and detect nucleic acids without specialized devices. We developed a RPAbased method for identifying Mycobacterium tuberculosis (MTB) by detecting mts90, a more specific target identified in our previous research. Different screening methods were employed for selecting a preferred primer pair of amplification, and probes were confirmed as very fast and reliable tools in the screening of potential primer candidates. The results showed that the mts90 RPA assay was very sensitive and capable of detecting 6 copies of recombinant plasmid containing mts90 sequence per reaction. The assay was specific for detecting MTB, as it did not identify the genomic DNA from other mycobacteria and pathogens. When applied to analyze clinical samples, including sputum, bronchoalveolar lavage fluid (BALF) and tissues, the mts90 RPA assay had a coincidence rate of $96.43 \%(27 / 28)$ compared to the Biochip test, which has been used in clinics for diagnosing TB. The mts90 RPA assay can be completed within 20 minutes at $39^{\circ} \mathrm{C}$ without thermal cycling; its simple operation and rapid detection suggest RPA-based MTB assays could be further developed for TB diagnosis in resource-poor settings.
\end{abstract}

\section{Introduction}

Tuberculosis (TB) is an infectious disease primarily caused by Mycobacterium tuberculosis complex (MTC) with a high morbidity and mortality, especially in ТB endemic countries and regions where healthcare resources are limited and inaccessible. The World Health Organization (WHO) "global TB report 2016" estimated that there were about 10.4 million new TB cases globally in 2015 and noted a 4.3 million gap between the estimated number of incident cases and notifications of new cases, ${ }^{1}$ indicating that there is a serious misdiagnosis in the

${ }^{a}$ Key Laboratory of Laboratory Medical Diagnostics Designated by the Ministry of Education, School of Laboratory Medicine, Chongqing Medical University, No. 1 Yixueyuan Road, Yuzhong District, Chongqing, 400016, P. R. China. E-mail: 100977@cqmu.edu.cn; Fax: +862368485992; Tel: +862368485239

${ }^{b}$ Department of Laboratory Medicine, The First Affiliated Hospital of Chongqing Medical University, Chongqing 400016, P. R. China

${ }^{\prime}$ Department of Respiratory Medicine, The First Affiliated Hospital of Chongqing Medical University, Chongqing 400016, P. R. China

${ }^{d}$ Institute of Life Sciences, Chongqing Medical University, Chongqing 400016, P. R. China

$\dagger$ Electronic supplementary information (ESI) available. See DOI: 10.1039/c7ra09999a current TB diagnosis and treatment. TB still poses a global health threat due to a lack of accurate diagnosis and timely treatment, coupled with HIV co-infection and the continued emergence of multidrug resistant or extreme drug-resistant strains. ${ }^{2-5}$ Focusing on global TB control, the United Nations put an end to the global ТВ epidemic as one of the 2030 sustainable development goals in the post-2015 era, ${ }^{6}$ and a strategy directed by WHO is to achieve a rapid and accurate diagnosis for more TB suspected patients. ${ }^{7}$

Conventional methods of TB diagnosis are sputum smear microscopy and culture. Smear microscopy is the primary test to diagnose TB. Many countries are phasing out this method because of its poor sensitivity and specificity. ${ }^{1,8}$ Bacterial culture is the current reference standard method; however, the requirement for laboratory capacities and up to 12 weeks to finalize the results and provide reports restrict its applications. ${ }^{1,9}$ Nucleic acid amplification tests (NAAT) aiming at a rapid and accurate detection of TB can avoid the limitations of conventional methods. ${ }^{10,11}$ The first rapid TB diagnostic method, Xpert MTB/RIF (Cepheid, USA), was endorsed by the WHO in 2010 and recommended as a preliminary screening test for individuals suspected of co-infection with HIV by 2015..$^{12,13}$ However, the Xpert MTB/RIF device demands specialized 
training of operators, stable electricity supply and a suitable working environment, ${ }^{\mathbf{1 4}}$ which are difficult for under-developed communities to afford. So it is worthwhile to develop a simple, rapid, sensitive and low-cost method for diagnosing TB to facilitate the need of healthcare systems in areas with a high TB burden. ${ }^{2,15}$

Unlike traditional PCR methods, isothermal amplification can amplify nucleic acids at a low incubation temperature without a precise thermal cycling, possessing the potential to develop point-of-care testing (POCT) application tools. ${ }^{16,17} \mathrm{~A}$ newly developed technique of isothermal nucleic acid amplification, recombinase polymerase amplification (RPA), mainly relies on three enzymes, i.e., recombinase, single-stranded DNA-binding protein (SSB) and strand displacement DNA polymerase, to complete testing in 20 minutes at an optimal working temperature, approximately $37{ }^{\circ} \mathrm{C}$ to $42{ }^{\circ} \mathrm{C} .{ }^{18}$ The protein-DNA complex formed by recombinase and primers can find homologous sequences in the target DNA, followed by a chain exchange reaction, and then the DNA synthesis is initiated by strand displacement polymerase. RPA can become a highly specific detection with a well-designed probe and RPA is ideally suited to field use with resource limits. ${ }^{18,19}$ Due to its diverse detection formats, high detection efficiency and adaptability, RPA has been widely applied in nucleic acid detection, such as infectious diseases diagnosis and cancer research. ${ }^{20-22}$ Particularly, RPA have been described for TB diagnosis by detecting MTC and nontuberculous mycobacteria (NTM). ${ }^{23-25}$

Mycobacterium tuberculosis (MTB) is an important member of MTC, accounting for $90 \%$ to $95 \%$ of the cases of human $\mathrm{TB},{ }^{26}$ and therefore it is crucial to detect MTB for the clinical diagnosis of TB. mtp40 is a frequently-used molecular target for identifying MTB; however, reports showed it is absent in some MTB strains and is also present in some other members of MTC. ${ }^{27,28}$ esat- 6 and cfp-10 are also used for detecting MTB, for which a RPA-based assay has been reported. ${ }^{29}$ But the esat- 6 and cfp-10 also exist in some NTM, such as Mycobacterium kansasii and Mycobacterium marinum..$^{30}$ In addition, IS6110 is a highly sensitive marker for detecting MTC, but some MTB strains cannot be identified due to lack of IS6110. ${ }^{31,32}$ In order to detect MTB more accurately and effectively, mts90 was identified as a more specific molecular diagnostic target of MTB in our previous study. ${ }^{33}$ Therefore, a method capable of sensitively detecting mts90 may help in improving the detection rate of TB and subsequently preventing TB transmission in the epidemic areas.

In this study, we established a real-time RPA method targeting mts90 for the rapid, sensitive and specific detection of MTB. The principle of this method is illustrated in Scheme 1. In addition, we evaluated the clinical usability of this assay by testing a variety of clinical samples.

\section{Materials and methods}

\subsection{Bacterium strains}

Mycobacterium tuberculosis H37Rv (ATCC27294) and Mycobacterium gastri (CMCC95006) were obtained from the National Institutes for Food and Drug Control (NIFDC, P. R. China). Mycobacterium bovis BCG and other pathogens, such as Staphylococcus aureus, Streptococcus pneumoniae D39, Pseudomonas aeruginosa and Escherichia coli, were from the Pathogenic Microbiology Laboratory of Chongqing Medical University (Chongqing, P. R. China). The genomic DNA of these bacteria was extracted according to the previous description. ${ }^{34}$

\subsection{Generation of reference plasmid}

According to the manufacturer's guidance, the plasmid standard of mts90 (2973968 to 2974057 nt of GenBank accession numbers CP009480.1) was constructed by a pMD19-T Vector Cloning Kit (TaKaRa). A fragment containing the mts90 region was ligated to pMD19-T vector, and then was transformed into cloning bacteria (DH5 $\alpha$ ). Plasmid DNA was extracted using the Plasmid Mini Kit (Omega Bio-tek) and verified by PCR as well as sequencing (TSINGKE Biological Technology, Chengdu, P. R. China). PCR reactions were performed in a $\mathrm{T} 100^{\mathrm{TM}}$ Thermal Cycler (Bio-Rad, USA) with the following protocol: $94{ }^{\circ} \mathrm{C}$ for $5 \mathrm{~min}$, followed by 30 cycles of $94{ }^{\circ} \mathrm{C}$ for $1 \mathrm{~min}, 63^{\circ} \mathrm{C}$ for $30 \mathrm{~s}$, $72{ }^{\circ} \mathrm{C}$ for $15 \mathrm{~s}$, and finally $72{ }^{\circ} \mathrm{C}$ for $10 \mathrm{~min}$. The DNA concentration was quantified by a NanoDrop $1000 \mathrm{UV} / \mathrm{Vis}$ spectrophotometer (Thermo Scientific, USA).

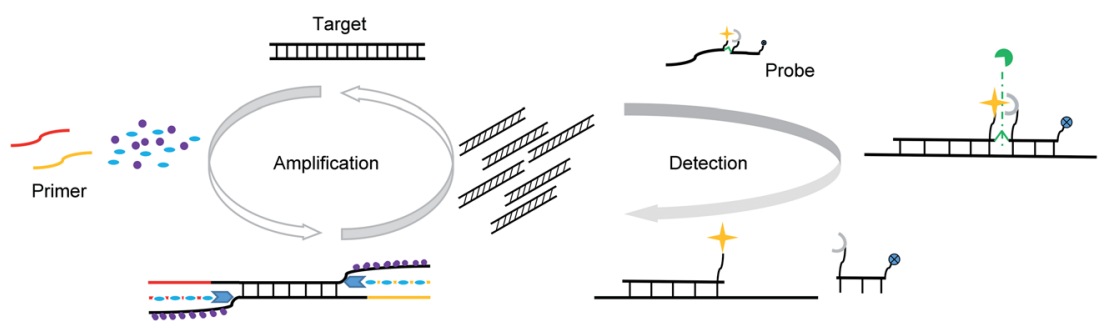

Real-time RPA reaction in $20 \mathrm{~min}$ at $39^{\circ} \mathrm{C}$
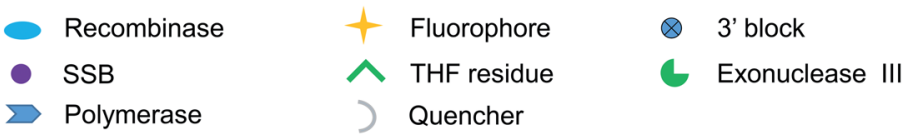

Scheme 1 Schematic illustration of real-time RPA performed on the amplification and detection of mts 90. 
Table 1 Primer sequences used for RPA and PCR assay

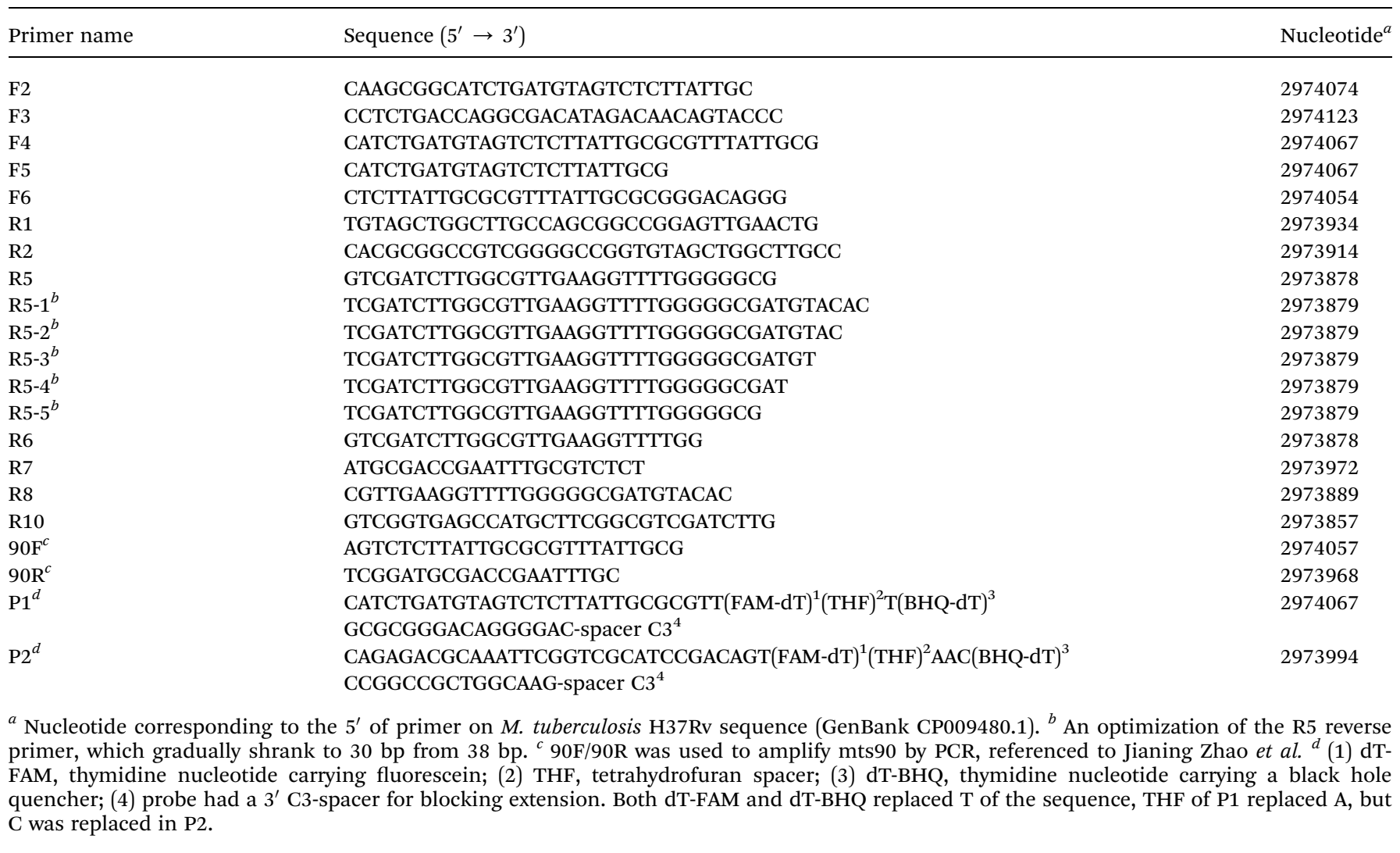

\subsection{RPA primer and probe design}

Primers and probes for the mts90 RPA assay were designed following the manufacturer's (TwistDx, Cambridge, UK) manual (https://www.twistdx.co.uk/en/support/rpa-assay-design-2).

Amplicons for the primer design were from conserved regions containing a nucleotide sequence of mts90. The screening of the primer candidates was accomplished via electrophoresis, fluorescent dye and probes for selecting a preferred primer set. All primers and probes (Table 1) were synthesized from Sangon Biotech (Shanghai, P. R. China).

\subsection{RPA assays}

RPA reactions analyzed by electrophoresis and fluorescent dye were performed on the TwistAmp ${ }^{\circledR}$ fpg kit (TwistDx, Cambridge, UK). Each reaction contained a $50 \mu \mathrm{L}$ mixture. A pre-mixture $(47.5 \mu \mathrm{L})$ was prepared firstly, including $2.1 \mu \mathrm{L}$ of the RPA primer pair (10 nM), $29.5 \mu \mathrm{L}$ of rehydration buffer, and $13.8 \mu \mathrm{L}$ of template and $\mathrm{ddH}_{2} \mathrm{O}$. Then the pre-mixture was diverted into the reaction tube to mix with TwistAmp fpg lyophilized enzyme pellets, and $2.5 \mu \mathrm{L}$ of magnesium acetate $(280 \mathrm{mM})$ were added to trigger the reaction immediately. The mixture was quickly placed in a $39^{\circ} \mathrm{C}$ water bath and incubated for at least $20 \mathrm{~min}$. Following purification by a High Pure PCR Product Purification Kit (Roche), its products were checked by agarose gel (2.5\%) electrophoresis. When monitored with fluorescent dye, $2.5 \mu \mathrm{L}$ of $20 \times$ EvaGreen ${ }^{\circledR}$ Dye (Biotum) were added in the pre-mixture up to $47.5 \mu \mathrm{L}$. After adding $2.5 \mu \mathrm{L}$ of magnesium acetate $(280 \mathrm{mM})$, RPA reactions were kept at $39^{\circ} \mathrm{C}$ with the Rotor Gene Q (Qiagen, Germany) and set to cycle readings every $20 \mathrm{~s}$.

RPA reactions coupled with a specific probe were carried out by the TwistAmp ${ }^{\circledR}$ exo kit (TwistDx, Cambridge, UK). The $47.5 \mu \mathrm{L}$ prereaction included $2.1 \mu \mathrm{L}$ of primer pair $(10 \mathrm{nM}), 0.6 \mu \mathrm{L}$ of probe (10 nM), $29.5 \mu \mathrm{L}$ of rehydration buffer, $13.2 \mu \mathrm{L}$ of template and $\mathrm{ddH}_{2} \mathrm{O}$. After adding $2.5 \mu \mathrm{L}$ of magnesium acetate $(280 \mathrm{mM})$, the mixture was rapidly transferred to the CFX Connect ${ }^{\mathrm{TM}}$ Real-time System (Bio-Rad, USA) and the reaction procedure was to incubate at $39^{\circ} \mathrm{C}$ and read fluorescence values every $20 \mathrm{~s}$ for $20 \mathrm{~min}$.

\subsection{Analytical sensitivity and specificity of mts90 RPA assay}

The reference plasmid was serially diluted to $6 \times 10^{5}, 6 \times 10^{4}, 6$ $\times 10^{3}, 6 \times 10^{2}, 6 \times 10^{1}$, and 6 copies per microliter, and these dilutions were used to determine the sensitivity of the mts90 RPA assay. To determine specificity, we detected DNA templates extracted from Mycobacterium tuberculosis $\mathrm{H} 37 \mathrm{Rv}$ (M. tuberculosis H37Rv), Mycobacterium bovis BCG (M. bovis BCG), Mycobacterium gastri (M. gastri), Escherichia coli (E. coli), Staphylococcus aureus (S. aureus), Streptococcus pneumoniae D39 (S. pneumoniae D39) and Pseudomonas aeruginosa (P. aeruginosa). $3 \mu \mathrm{L}$ of genomic DNA of each strain was added into the RPA reactions.

\subsection{Clinical samples for the assessment of the mts90 RPA assay application}

A total of 28 clinical samples from the First Affiliated Hospital of Chongqing Medical University (Chongqing, P. R. China) were used 
to evaluate the mts90 RPA assay. This protocol was approved by the Clinical Research Ethics Committee of Chongqing Medical University. These clinical samples were identified as MTC positive by a Biochip test (CapitalBio Corporation, Beijing, P. R. China). Pre-treatment for the digestion and decontamination of the clinical samples was performed by using a reference $\mathrm{NaOH}-\mathrm{Na}$ citrate standard method, ${ }^{35}$ and then the supernatant was discarded after centrifugation, and the pellet was washed and resuspended repeatedly with sterile PBS. ${ }^{36}$ The DNA of the clinical samples was obtained by using the nucleic acid extraction reagents in the Mycobacteria Identification Array Kit (CapitalBio Corporation, Beijing, P. R. China). $2.5 \mu \mathrm{L}$ of the DNA of each sample were used as the template in the RPA reactions.

\section{Results}

\subsection{Design of mts90 RPA assay}

To select a preferred primer pair, we used different methods to screen for the optimal primer pair in corresponding candidate primers (Table 1) and conducted RPA reactions for the reference plasmid. The results of electrophoresis showed that the primer pair of F3/R2 had the best amplification effect among 5 pairs of primers (Fig. S1 $\dagger$ ). Monitoring the performance of different primers in real time is a method that can minimize the risk of cross-contamination caused by aerosols when opening lids. By adding fluorescent dye (EvaGreen ${ }^{\circledR}$ Dye) to perform real-time detection, only one pair (F3/R5) of 4 primer sets was selected for acquiring the most obvious difference between a positive amplification and negative control (Fig. S1B $\dagger$ ). In addition, two probes were designed to test different primer pairs. The results demonstrated that the combination of probe P2 and primer pair F3/R10 produced the highest fluorescence signal among 7 pairs of primers (Fig. 1A). In order to determine the suitable one for further electrophoresis analysis, three pairs of primers selected above were further verified with genomic DNA from $M$. tuberculosis H37Rv. The products were viewed on a $2.5 \%$ agarose gel after purification. The band of the F3/R10 positive lane showed the highest amplification performance (Fig. 1B). Therefore, probe P2 and primer pair F3/R10 were used for subsequent experiments.

\subsection{Sensitivity analysis of mts90 RPA assay}

The sensitivity of the mts90 RPA assay was investigated by detecting a serial dilution of the reference plasmid. The results showed that the 10-fold dilutions of plasmid from $6 \times 10^{5}$ to $6 \times$ $10^{\circ}$ copies per reaction could produce an amplification signal within 20 minutes (Fig. 2), and the fluorescence intensity of 6 copies per reaction was approximately $200 \mathrm{RFU}$, which was significantly higher than that of the background. It was proved by repeated tests $(n \geq 3)$ on different concentrations that the sensitivity and repeatability of the mts90 RPA assay were valid (Table 2). Six (6)-copy preparation was amplified in 5 of $5(100 \%)$, and the time needed for a positive amplification signal was after about 8 minutes (Fig. 3). The results above demonstrated that the sensitivity of this assay was as low as 6 copies per reaction.

\subsection{Specificity of mts90 RPA assay}

To analyze the specificity of the mts90 RPA assay for MTB, we detected the genomic DNA extracted from seven strains. As

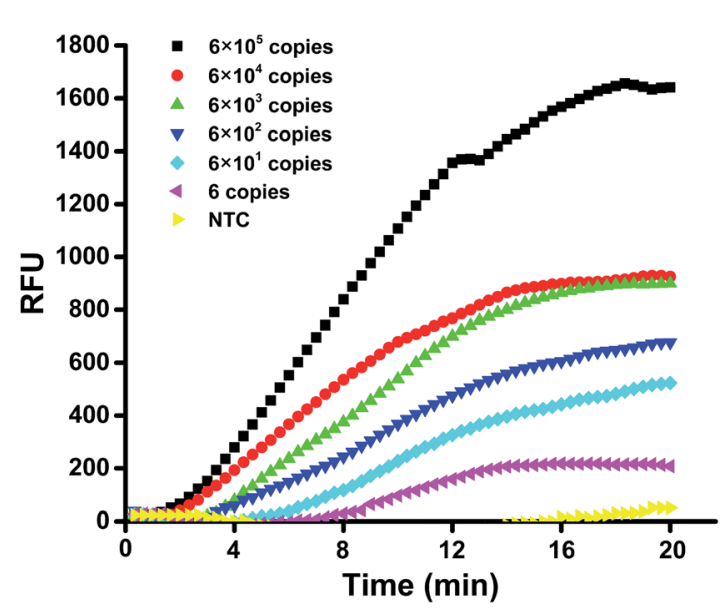

Fig. 2 Real-time RPA amplification of mts90 for a sensitivity analysis. Fluorescence development via real-time detection using a dilution range of $6 \times 10^{5}$ to $6 \times 10^{0}$ copies per $\mu \mathrm{L}$ of a given reference plasmid. The template of different concentrations for each reaction was 1 microliter. NTC (no template control).
A

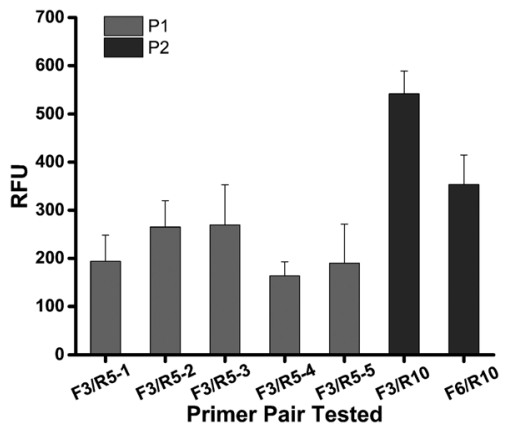

B

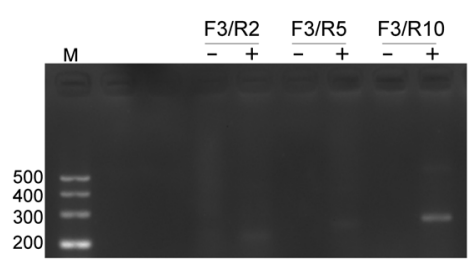

Fig. 1 The screening of different primer pairs. (A) Different primer pairs coupled with the corresponding probe were tested with 60 copies of plasmid DNA for each reaction. The combination of P2 and F3/R10 was higher than others in the Relative Fluorescence Unit (RFU), $n=3$. (B) Primer pairs (F3/R2; F3/R5; F3/R10) selected by electrophoresis, fluorescent dye and probes were verified by $2.5 \%$ agarose gel electrophoresis. The template was $3 \mu \mathrm{L}$ of genomic DNA of M. tuberculosis H37Rv. The primer set of F3/R10 obtained the brightest electrophoresis strap. Lane M, 500 bp DNA ladder. - (no template control); + (positive reaction). 
Table 2 Sensitivity of mts90 RPA assay ${ }^{a}$

\begin{tabular}{ll}
\hline mts90 RPA (copies per reaction) & Positive reactions/replicates \\
\hline $1.5 \times 10^{6}$ & $3 / 3$ \\
$1.5 \times 10^{5}$ & $3 / 3$ \\
$1.5 \times 10^{4}$ & $3 / 3$ \\
$1.5 \times 10^{3}$ & $3 / 3$ \\
$1.5 \times 10^{2}$ & $3 / 3$ \\
$1.5 \times 10^{1}$ & $4 / 4$ \\
6 & $5 / 5$ \\
0 & $0 / 3$
\end{tabular}

${ }^{a}$ The data used for the sensitivity analysis of mts90 RPA assay were obtained from testing on the plasmid. The sensitivity was as low as 6 copies per reaction for a $100 \%$ positive rate of detection $(5 / 5)$.

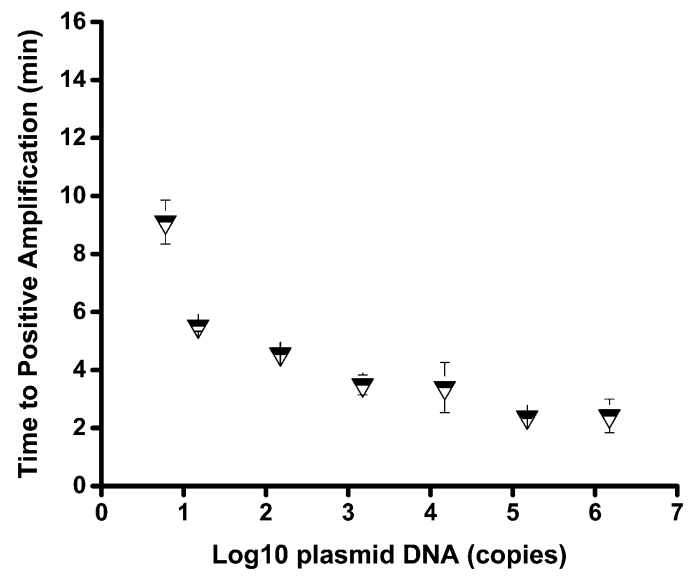

Fig. 3 The time to positive amplification for different concentrations of plasmid. The concentrations ranged from $1.5 \times 10^{6}$ to $1.5 \times 10^{1}$ copies per reaction and were successfully amplified within $8 \mathrm{~min}$, and 6 copies per reaction could get a positive amplification signal after about 8 min, $n \geq 3$.

expected, only M. tuberculosis $\mathrm{H} 37 \mathrm{Rv}$ was found to be positive with the positive control of the mts90 plasmid in real-time detection (Fig. 4A). Although M. bovis BCG and M. gastri are members of MTC, the strains tested were negative. Similarly, the remaining other pathogenic bacteria did not produce any
Table 3 The results of clinical samples detected by the mts90-RPA assay

\begin{tabular}{llrlll}
\hline Sample \# & Sample type & RPA(TT $^{a}$ & Sample \# & Sample type & RPA(TT) \\
\hline 1 & Sputum & 3.81 & 15 & Sputum & 12.64 \\
2 & Sputum & 7.60 & 16 & BALF & 9.82 \\
3 & Sputum & 19.64 & 17 & BALF & 7.90 \\
4 & Sputum & 4.36 & 18 & BALF & 8.73 \\
5 & Sputum & 9.17 & 19 & BALF & 10.20 \\
6 & Sputum & 9.78 & 20 & BALF & 16.86 \\
7 & Sputum & 11.67 & 21 & BALF & 17.97 \\
8 & Sputum & 11.48 & 22 & BALF & 11.66 \\
9 & Sputum & 8.34 & 23 & BALF & 11.79 \\
10 & Sputum & 15.28 & $24^{b}$ & BALF & NG \\
11 & Sputum & 15.00 & 25 & Tissue & 16.61 \\
12 & Sputum & 7.62 & 26 & Tissue & 15.22 \\
13 & Sputum & 12.49 & 27 & Tissue & 19.28 \\
14 & Sputum & 10.06 & 28 & Tissue & 13.23
\end{tabular}

${ }^{a}$ TT, threshold time in minutes. TT $(\mathrm{min})=(\mathrm{CT}$ values $\times 20 \mathrm{~s}$ per cycle $) /$ 60, CT was calculated by using Bio-Rad CFX Manager software 3.1. ${ }^{b}$ Sample \# 24, the sample of BALF, was detected in the negative by the mts90 RPA assay, NG (negative).

positive amplification signal. Furthermore, the results analyzed by electrophoresis were consistent with the above. The bands of M. tuberculosis $\mathrm{H} 37 \mathrm{Rv}$ and plasmid are shown at the same size (Fig. 4B). Thus, the mts90 RPA assay established for detecting MTB had the desired specificity.

\subsection{Performance evaluation of mts90 RPA assay with clinical samples}

The performance of the mts90 RPA assay was evaluated by testing clinical samples. A total of 28 clinical samples were collected that were identified as positive by a Biochip test, including sputum (15), bronchoalveolar lavage fluid (BALF - 9) and lung tissue (4). The mts90 RPA assay could successfully detect MTB from the clinical samples within 20 minutes (Table 3 ). There was a $100 \%$ detection rate when testing samples of sputum and tissue (15/15 and 4/4, respectively). Although for the samples of BALF, 8 in 9 cases were detected positive by the mts90 RPA assay and one of them was negative, the total coincidence rate was high, up to $96.43 \%(27 / 28)$ (Table 4$)$.

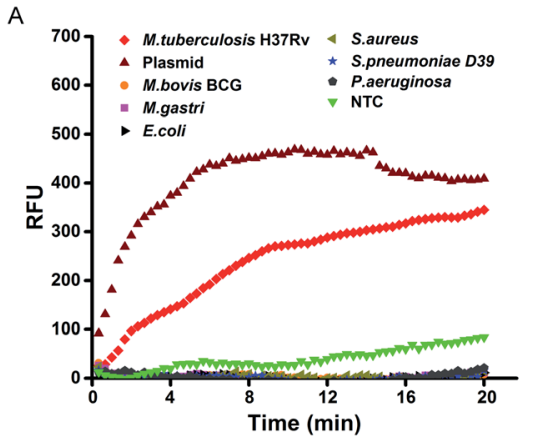

B

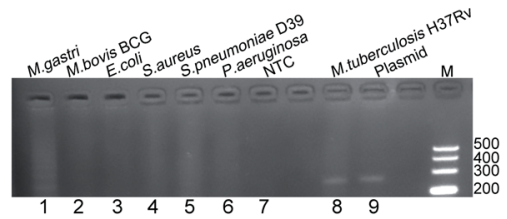

Fig. 4 Analytical specificity of mts90 RPA assay by real-time detection and gel. (A) Real-time RPA amplification of genomic DNA extracted from a group of representative strains. Plasmid, as a positive control. (B) The products of RPA reactions were analyzed by electrophoresis. Lane (1-9), M. gastri; M. bovis BCG; E. coli; S. aureus; S. pneumoniae D39; P. aeruginosa; NTC; M. tuberculosis H37Rv; plasmid; lane (M), 500 bp DNA ladder. 
Table 4 Evaluation performance of mts90 RPA assay by detecting clinical samples ${ }^{a}$

\begin{tabular}{lll}
\hline Sample type & Case & Coincidence rate $(n / N)$ \\
\hline Sputum & 15 & $100 \%(15 / 15)$ \\
$\begin{array}{l}\text { BALF (bronchoalveolar } \\
\text { lavage fluid) }\end{array}$ & 9 & $88.89 \%(8 / 9)$ \\
$\begin{array}{l}\text { Tissue (lung) } \\
\text { Total }\end{array}$ & 4 & $100 \%(4 / 4)$ \\
& 28 & $96.43 \%(27 / 28)$
\end{tabular}

${ }^{a}$ All the clinical samples were identified as MTC positive by Biochip test (CapitalBio Corporation). The results showed that these samples were detected totally in 27 of $28(96.43 \%)$ positive cases by mts90 RPA assay, $n$ (number of positive amplifications by real-time RPA detection), $N$ (total number of samples).

\section{Discussion}

A rapid and sensitive mts90 RPA assay for detecting MTB was established and it was successfully applied to detect clinical samples. According to the manufacturer's guidelines, primer selection is critical to the development of RPA methods, and it is necessary to use a long primer (30-35 bases) to obtain the desired amplification effect. However, based on a recent review paper, some published RPA studies used primers below 30 bases or over 35 bases, ${ }^{37}$ showing some differences from the guidelines. Therefore, different primer lengths (22-38 bases) were designed and tested in our experiments.

The primer set of F3/R2 (32/35 bases) was selected based on electrophoresis analysis. F3/R5 (R5, 31 bases) was selected by fluorescent dye (EvaGreen $₫$ Dye); while F3/R10 (R10, 31 bases) was screened using well-designed probes. The lengths of the primers obtained from the above screening methods were within the recommended range, which explains that the appropriately long primers might be preferred for RPA amplification. Certainly, a RPA reaction executed at a single temperature $\left(37^{\circ} \mathrm{C}\right)$ within 20 minutes also relies on the initial template concentration and amplicon size..$^{38}$ In this study, we spent much of the time for the purification of RPA products before electrophoresis analysis, and it remains a challenge for analytical specificity using intercalating dye to monitor real-time fluorescence; however, probes could rapidly generate comparative data after 16 minutes and improve the specificity among the screening. Moreover, the primer set of F3/R10 selected by the probes showed the best amplification effect when tested with genomic DNA of M. tuberculosis H37Rv. Thus, we confirmed that it is more efficient and reliable to use probes for selecting a preferred primer pair in the development of RPA methods.

The sensitivity of the mts90 RPA assay was high with only 6 copies per reaction for detecting the reference plasmid. The sensitivity was slightly higher than that of the TaqMan qPCR method developed previously; its sensitivity was 10 copies of plasmid. ${ }^{33}$ Fig. 3 shows that the time needed to reach a positive amplification depends on the input concentrations of the plasmid, but this proportional relation was not strict. As for the analytical specificity of the mts90 RPA assay, it could successfully detect M. tuberculosis $\mathrm{H} 37 \mathrm{Rv}$ but not M. bovis BCG, M. gastri and other pathogens. The probe (P2) used for the mts90 RPA assay was 52 oligonucleotides in length with homologous sequences of mts90, which ensured that the assay was capable of specifically detecting MTB in the RPA reactions. The structure of probe $\mathrm{P} 2$ was also analyzed online (http://www.nupack.org/), seen in Fig. S2B. $\dagger$

The clinical performance of the mts90 RPA assay was evaluated by detecting clinical samples. The results showed that the accuracy of this assay was $96.43 \%(27 / 28)$, with only a unique case of BALF that tested inconsistently with clinical results. These specimens were identified as MTC positive using a Biochip system that is currently the only approved one certified by the China Food and Drug Administration (CFDA) for the rapid identification of mycobacteria. The Biochip test can identify MTC and 16 common NTMs; ${ }^{39}$ but it cannot identify specific types of MTC. Therefore, the sample detected as negative by the mts90 RPA assay might be a strain other than MTB within MTC; thus, further sequencing is required. However, the clinical utility of this assay requires more clinical samples to be further evaluated.

Compared to the Biochip test, our real-time RPA assay only requires basic operation technical skills and typically 20 minutes of reaction time, which is much simpler than the complicated testing procedures of about 6 hours for the Biochip system..$^{39,40}$ Although other well-established techniques for the diagnosis of TB by detecting nucleic acids exist, RPA has its own special advantages for POCT diagnosis in particular. Unlike many PCR assays (such as Xpert MTB/RIF), RPA has the potential for miniaturization and is cost effective, as it could complete its amplification and detection with existing facilities. Furthermore, fluorescent detection is really not reliant on costly special equipment. A simple battery powered device is also feasible. ${ }^{41,42}$ The loop-mediated isothermal amplification test for TB (TB-LAMP) reviewed and recommended by WHO in 2016 is a manual test that takes less than 1 hour and its results can be read with the naked eye under ultraviolet light. ${ }^{43}$ However, RPA further reduces the testing time and only two primers are necessary, which is simpler than the four to six primers required for the LAMP reactions. ${ }^{44}$ Besides, RPA requires a lower energy and may proceed at temperatures between $25^{\circ} \mathrm{C}$ and $42{ }^{\circ} \mathrm{C}$ compared with around $65{ }^{\circ} \mathrm{C}$ for LAMP..$^{18,45}$ More significantly, most required reagents form as a freeze-dried pellet to simplify the testing and all the reagents involved in the RPA reactions are cold-chain independent. ${ }^{46}$ Overall, RPA is more suitable for healthcare in remote parts of the globe.

\section{Conclusion}

In summary, the mts90 RPA assay showed a high sensitivity and specificity for detecting MTB, and the assay was simple to operate, as well as quick with a reaction that could be completed within 20 minutes, greatly improving the detection efficiency. Therefore, the mts90 RPA assay developed in this study can be a complement to the existing nucleic acid diagnostic methods of TB. Further in-depth studies may be necessary to design a simpler readout method for the mts90 RPA assay and apply the assay to diagnose ТВ in low-income and TB high-burden areas. 


\section{Conflicts of interest}

The authors declare no conflict of interest.

\section{Acknowledgements}

This study was supported by Society and Livelihood Foundation of Chongqing (grant No. cstc2015shmszx120098).

\section{References}

1 W. H. Organization, Global tuberculosis report 2016, World Health Organization, 2016.

2 M. Pai and M. Schito, J. Infect. Dis., 2015, 211(suppl. 2), S21S28.

3 N. R. Gandhi, P. Nunn, K. Dheda, H. S. Schaaf, M. Zignol, D. van Soolingen, P. Jensen and J. Bayona, Lancet, 2010, 375, 1830-1843.

4 G. B. Migliori, A. Matteelli, D. Cirillo and M. Pai, Canadian Journal of Infectious Diseases and Medical Microbiology, 2016, 19, 169.

5 J. Bruchfeld, M. Correia-Neves and G. Kallenius, Cold Spring Harbor Perspect. Med., 2015, 5, a017871.

6 G. A. UN, Transforming our world: The 2030 agenda for sustainable development, General Assembly of the United Nations, 2015.

7 W. H. Organization, Draft Report of the Sixty-Seventh World Health Assembly on Global Strategy and Targets for Tuberculosis Prevention, Care and Control after 2015, World Health Organization, 2015.

8 S. D. Lawn and A. I. Zumla, Lancet, 2011, 378, 57-72.

9 S. Asmar and M. Drancourt, Front. Microbiol., 2015, 6, 1184.

10 Y. Derese, E. Hailu, T. Assefa, Y. Bekele, A. Mihret, A. Aseffa, J. Hussien, I. Ali and M. Abebe, J. Infect. Dev. Countries, 2012, 6, 53-57.

11 C. C. Boehme, M. P. Nicol, P. Nabeta, J. S. Michael, E. Gotuzzo, R. Tahirli, T. G. Ma, R. Blakemore, W. Worodria and C. Gray, Lancet, 2011, 377, 1495-1505.

12 C. C. Boehme, P. Nabeta, D. Hillemann, M. P. Nicol, S. Shenai, F. Krapp, J. Allen, R. Tahirli, R. Blakemore, R. Rustomjee, A. Milovic, M. Jones, S. M. O'Brien, D. H. Persing, S. Ruesch-Gerdes, E. Gotuzzo, C. Rodrigues, D. Alland and M. D. Perkins, N. Engl. J. Med., 2010, 363, 1005-1015.

13 W. H. Organization, Global tuberculosis report 2015, World Health Organization, 2015.

14 C. M. Denkinger, I. Nicolau, A. Ramsay, P. Chedore and M. Pai, Eur. Respir. J., 2013, 42, 544-547.

15 S. A. Cheon, H. H. Cho, J. Kim, J. Lee, H. J. Kim and T. J. Park, J. Microbiol. Methods, 2016, 123, 51-61.

16 L. Yan, J. Zhou, Y. Zheng, A. S. Gamson, B. T. Roembke, S. Nakayama and H. O. Sintim, Mol. BioSyst., 2014, 10, 970-1003.

17 J. Li and J. Macdonald, Biosens. Bioelectron., 2015, 64, 196211.

18 O. Piepenburg, C. H. Williams, D. L. Stemple and N. A. Armes, PLoS Biol., 2006, 4, e204.
19 Z. A. Crannell, B. Rohrman and R. Richardskortum, PLoS One, 2014, 9, e112146.

20 D. S. Boyle, D. A. Lehman, L. Lillis, D. Peterson, M. Singhal, N. Armes, M. Parker, O. Piepenburg and J. Overbaugh, mBio, 2013, 4, 49-52.

21 M. D. Moore and L. A. Jaykus, Sci. Rep., 2017, 7, 40244.

22 Y. Liu, T. Lei, Z. Liu, Y. Kuang, J. Lyu and Q. Wang, Int. J. Mol. Sci., 2016, 17, 792.

23 D. S. Boyle, R. McNerney, H. Teng Low, B. T. Leader, A. C. Perez-Osorio, J. C. Meyer, D. M. O'Sullivan, D. G. Brooks, O. Piepenburg and M. S. Forrest, PLoS One, 2014, 9, e103091.

24 Q. Ma, H. Liu, F. Ye, G. Xiang, W. Shan and W. Xing, Mol. Cell. Probes, 2017, DOI: 10.1016/j.mcp.2017.08.004.

25 S. Hansen, J. Schafer, K. Fechner, C. P. Czerny and A. Abd El Wahed, PLoS One, 2016, 11, e0168733.

26 L. M. Parsons, R. Brosch, S. T. Cole, A. Somoskovi, A. Loder, G. Bretzel, D. Van Soolingen, Y. M. Hale and M. Salfinger, J. Clin. Microbiol., 2002, 40, 2339-2345.

27 A. Weil, B. B. Plikaytis, W. R. Butler, C. L. Woodley and T. M. Shinnick, J. Clin. Microbiol., 1996, 34, 2309-2311.

28 E. Liébana, A. Aranaz, B. Francis and D. Cousins, J. Clin. Microbiol., 1996, 34, 933-938.

29 B. Y. C. Ng, E. J. H. Wee, N. P. West and M. Trau, Sci. Rep., 2015, 5, 15027.

30 S. M. Arend, K. E. van Meijgaarden, K. de Boer, E. C. de Palou, D. van Soolingen, T. H. Ottenhoff and J. T. van Dissel, J. Infect. Dis., 2002, 186, 1797-1807.

31 S. D. Van, P. E. de Haas, P. W. Hermans, P. M. Groenen and J. D. van Embden, J. Clin. Microbiol., 1993, 31, 1987.

32 M. N. Huyen, E. W. Tiemersma, K. Kremer, P. de Haas, N. T. Lan, T. N. Buu, C. Sola, F. G. Cobelens and D. van Soolingen, International Journal of Tuberculosis and Lung Disease, 2013, 17, 1479-1485.

33 J. Zhao, Y. Wang, D. Li, J. Liu, X. Zhang, Y. He, H. Wang, J. Cao, Y. Yin and W. Xu, World J. Microbiol. Biotechnol., 2014, 30, 2189-2197.

34 C. Bouakaze, C. Keyser, S. J. D. Martino, W. Sougakoff, N. Veziris, H. Dabernat and B. Ludes, J. Clin. Microbiol., 2010, 48, 1758.

35 P. T. Kent and G. P. Kubica, Public health mycobacteriology. A guide to the level III laboratory, US Department of Health and Human Services, 1985.

36 D. I. Gomez, C. S. Mullin, F. Mora-Guzmán, J. G. CrespoSolis, S. P. Fisher-Hoch, J. B. Mccormick and B. I. Restrepo, Tuberculosis, 2011, 91, S43-S48.

37 R. K. Daher, G. Stewart, M. Boissinot and M. G. Bergeron, Clin. Chem., 2016, 62, 947-958.

38 G. A. Hill-Cawthorne, L. O. Hudson, M. F. El Ghany, O. Piepenburg, M. Nair, A. Dodgson, M. S. Forrest, T. G. Clark and A. Pain, PLoS One, 2014, 9, e101419.

39 L. Zhu, G. Jiang, S. Wang, C. Wang, Q. Li, H. Yu, Y. Zhou, B. Zhao, H. Huang, W. Xing, K. Mitchelson, J. Cheng, Y. Zhao and Y. Guo, J. Clin. Microbiol., 2010, 48, 3654-3660. 40 Y. Pang, Y. Zhou, S. Wang, Y. Tan, J. Yue, B. Zhao, L. Wang, Y. Zhao and K. M. Kam, International Journal of Tuberculosis and Lung Disease, 2011, 15, 1680-1685. 
41 A. E. W. Ahmed, W. Manfred and F. T. Hufert, J. Clin. Virol., 2015, 69, 16-21.

42 D. Mondal, P. Ghosh, M. A. A. Khan, F. Hossain, S. BöhlkenFascher, G. Matlashewski, A. Kroeger, P. Olliaro and A. A. E. Wahed, Parasites Vectors, 2016, 9, 281.

$43 \mathrm{~W}$. H. Organization, The Use of Loop-Mediated Isothermal Amplification (TB-LAMP) for the Diagnosis of Pulmonary Tuberculosis: Policy Guidance, World Health Organization, 2016.

44 K. Nagamine, T. Hase and T. Notomi, Mol. Cell. Probes, 2002, 16, 223.
45 T. Notomi, H. Okayama, H. Masubuchi, T. Yonekawa, K. Watanabe, N. Amino and T. Hase, Nucleic Acids Res., 2000, 28, E63.

46 O. Faye, O. Faye, B. Soropogui, P. Patel, A. A. E. Wahed, C. Loucoubar, G. Fall, D. Kiory, N. F. Magassouba, S. Keita, M. K. Kondé, A. A. Diallo, L. Koivogui, H. Karlberg, A. Mirazimi, O. Nentwich, O. Piepenburg, M. Niedrig, M. Weidmann and A. A. Sall, Eurosurveillance, 2015, 20, 30053. 